\title{
Ampicillin-resistant Haemophilus influenzae isolates in Geneva: serotype, antimicrobial susceptibility, and $\beta$-lactam resistance mechanisms
}

\author{
A. Cherkaoui $^{1}$ - S. M. Diene ${ }^{2} \cdot$ S. Emonet ${ }^{1} \cdot$ G. Renzi $^{1} \cdot$ P. Francois $^{2} \cdot$ J. Schrenzel $^{1,2}$
}

Received: 26 March 2015 / Accepted: 22 June 2015 / Published online: 18 July 2015

(C) Springer-Verlag Berlin Heidelberg 2015

\begin{abstract}
The purpose of this study was to analyze the molecular mechanisms of ampicillin-resistant Haemophilus influenzae isolated in Geneva, Switzerland. We investigated the association between specific patterns of amino acid substitutions in penicillin-binding protein 3 (with or without $\beta$-lactamase production) and $\beta$-lactam susceptibility. Another main focus for this study was to compare the accuracy of disk diffusion and Etest methods to detect resistance to ampicillin and amoxicillin/ clavulanic acid. The antibiotic susceptibility to $\beta$-lactam antibiotics of $124 \mathrm{H}$. influenzae isolates was determined by disk diffusion and Etest methods, and interpreted by European Committee on Antimicrobial Susceptibility Testing (EUCAST) and Clinical and Laboratory Standards Institute (CLSI) breakpoints. Alterations in PBP3 were investigated by sequencing the ftsI gene. Of the 124 clinical isolates analyzed, ampicillin resistance was found in $36 \%$ (45 out of 124). The rate of resistance to amoxicillin/clavulanic acid was $9 \%$ and $0.8 \%$, using EUCAST and CLSI breakpoints respectively. For the 78 $\beta$-lactamase negative ampicillin-susceptible (BLNAS) isolates for which the Etest method indicated a high degree of susceptibility (MIC $\leq 1 \mathrm{mg} / \mathrm{L}$ ), the disk diffusion method revealed resistance to ampicillin and amoxicillin/
\end{abstract}

A. Cherkaoui

abdessalam.cherkaoui@hcuge.ch

1 Bacteriology Laboratory, Division of Laboratory Medicine, Department of Genetics and Laboratory Medicine, Geneva University Hospitals, 4 rue Gabrielle-Perret-Gentil, 1205 Geneva, Switzerland

2 Genomic Research Laboratory, Service of Infectious Diseases, Geneva University Hospitals, 4 rue Gabrielle-Perret-Gentil, 1205 Geneva, Switzerland clavulanic acid in 33 cases (42\%). Most common amino acid substitutions were Asn526Lys and Val547Ile, followed by Asp569Ser, Ala502Val, Asp350Asn, Met377Ile, Ile449Val, and Arg517His. The patterns observed were classified into six groups (IIa, IIb, IIc, IId, III-like, and miscellaneous). Continued characterization of both invasive and respiratory $H$. influenzae isolates is necessary in order to observe changes in the microbiology and epidemiology of this pathogen that could lead to clinical failure when treated by empirical antibiotic therapy.

\section{Introduction}

Resistance to ampicillin in Haemophilus influenzae was described at the beginning of the 1970s [1,2]. $\beta$-Lactams have long been used to treat $H$. influenzae infections, but resistance has emerged and disseminated. Ampicillin resistance in $H$. influenzae is now globally widespread, with incidence rates varying from 8 to $30 \%$ in different European countries and North America to more than $50 \%$ in some Eastern Asian countries [3]. Two $\beta$-lactam resistance mechanisms have been described. One involves enzymatic hydrolysis of $\beta$-lactam by TEM-1 or ROB-1 $\beta$-lactamases, and such isolates are denoted $\beta$-lactamase-positive ampicillin-resistant (BLPAR). The other involves decreased $\beta$-lactam affinity for penicillin binding protein 3 (PBP3) owing to alteration in the ftsI gene $[4,5]$. The nomenclature of antimicrobial resistant $H$. influenzae is complex. The commonly used term, $\beta$-lactamase-negative ampicillin-resistant (BLNAR), is used for isolates with ampicillin resistance, but no evidence of $\beta$-lactamase production [6]. Ampicillin resistance in such isolates was due to key mutations in the ftsI gene (encoding penicillin-binding protein 3 [PBP-3]), which lowered the affinity for $\beta$-lactam [7]. However, some isolates had such mutations, but were not 
ampicillin-resistant according to phenotype testing. Isolates with key mutations in PBP-3 regardless of resistance phenotype are designated as genomic BLNAR (gBLNAR) [2]. The commonly used term, $\beta$-lactamase positive, amoxicillin/ clavulanic acid-resistant (BLPACR), is used for isolates with amoxicillin/clavulanic acid resistance and evidence of $\beta$ lactamase production [7]. Such isolates were found to possess both the $\beta$-lactamase gene and key mutations in the ftsI gene [8]. Some isolates had such a profile, but were not amoxicillin/ clavulanic acid-resistant, according to phenotype testing. These isolates are designated genomic BLPACR (gBLPACR) [2]. Resistance due to altered penicillin-binding proteins has emerged as an important mechanism of $\beta$-lactam resistance in many bacterial pathogens, including nontypeable $H$. influenzae (NTHi). Over the past decade many countries have documented a rapid increase in the prevalence of BLNAR NTHi isolates, particularly among respiratory tract isolates [9]. As a result, genotypically defined BLNAR (gBLNAR) isolates account for 15-30\% of all NTHi isolates in Australia, in the USA, and in Europe, and an alarming rate of $50 \%$ in Japan [10, 11].

Haemophilus influenzae is an important bacterial pathogen that causes community-acquired respiratory tract infections, such as acute otitis media, acute sinusitis, acute exacerbations of chronic bronchitis, and community-acquired pneumonia $[1,12]$. Owing to the severity of these infections and the difficulties in determining the bacterial etiology and the antimicrobial susceptibility, treatment is often empirical, usually consisting of orally administered agents. The choice of empiric antimicrobial chemotherapy is guided by the clinical presentation, the severity of the infection, and epidemiological data that include the causative organisms and their local susceptibility to antimicrobial agents [13]. Accurate molecular characterization of $H$. influenzae clinical isolates, therefore, appears very important in monitoring and understanding changes in the epidemiology of these bacteria. High-quality epidemiological data will help to improve therapeutic guidelines for infections due to $H$. influenzae.

The present study had three main objectives:

1. To determine the susceptibility to $\beta$-lactam antibiotics of a collection of 124 clinical isolates of $H$. influenzae, according to European Committee on Antimicrobial Susceptibility Testing (EUCAST) and Clinical and Laboratory Standards Institute (CLSI) criteria.

2. To analyze the molecular mechanisms of ampicillinresistant $H$. influenzae, with special emphasis on BLNAR isolates.

3. To compare the disk diffusion and Etest methods for their accuracy at detecting resistance to ampicillin and amoxicillin/clavulanic acid according to EUCAST criteria.

\section{Materials and methods}

\section{Strains and growth conditions}

This work examined $124 H$. influenzae strains collected between 2009 and 2014. These isolates were chosen according to their different ampicillin MICs. All strains were stored at $-80{ }^{\circ} \mathrm{C}$ in skim milk with $15 \%$ glycerol. $H$. influenzae were cultured on chocolate agar supplemented with polyvitex (bioMérieux SA, Geneva, Switzerland) and incubated at $35{ }^{\circ} \mathrm{C}$ for $18-24 \mathrm{~h}$ in a humid atmosphere containing $5 \%$ $\mathrm{CO}_{2}$. Identification of the $124 \mathrm{H}$. influenzae isolates was confirmed using MALDI TOF/MS (Bruker Daltonics, Bremen, Germany) [14-16], according to the manufacturers' instructions. The MALDI TOF/MS score values ranged between 2.005 and 2.427 .

\section{Antimicrobial susceptibility testing}

Antimicrobial susceptibility testing was performed using both the Etest and the disk diffusion method according to EUCAST guidelines. The minimum inhibitory concentration (MIC) for ampicillin, amoxicillin/clavulanic acid, cefuroxime, and piperacillin/tazobactam were determined using the Etest method (bioMérieux SA). The inoculum suspension was prepared by selecting several colonies from overnight growth (16-24 h of incubation) on chocolate agar plates supplemented with polyvitex with a cotton swab and suspending the colonies in sterile saline $(0.85 \% \mathrm{NaCl} \mathrm{w} / \mathrm{v})$ to the density of a McFarland 0.5 standard. The inoculum was spread over the entire surface of the Mueller-Hinton agar plate $(+5 \%$ defibrinated horse blood and $20 \mathrm{mg} / \mathrm{L} \beta$-NAD) by swabbing in three directions, and incubated in a humid atmosphere containing $5 \% \mathrm{CO}_{2}, 35$ $\pm 1{ }^{\circ} \mathrm{C}$, for $18 \pm 2 \mathrm{~h}$. Disk diffusion was performed according to the EUCAST guidelines. $\beta$-lactamase production was screened by the chromogenic cephalosporin assay using a nitrocefin disk (Becton Dickinson AG, Allschwil, Switzerland). For quality control we used $H$. influenzae ATCC 49766 and H. influenzae NCTC 8468.

\section{Screening for ampicillin-resistant determinants}

The presence of the $\beta$-lactamase-encoding $b l a_{\mathrm{TEM}-1}$ gene was investigated in all 124 isolates by PCR using the following primers and probe:

$$
\begin{aligned}
& \text { TEM-1 }{ }^{\text {frw }} \text { (5'-GTGCGGTATTATCCCGTGTTG-3') } \\
& \text { TEM-1 }^{\text {rev }}\left(5^{\prime}\right. \text { '-GTATGCGGCGACCGAGTT-3') } \\
& \text { TEM-1 }^{\text {MGB PROBE }} \text { (5'-CGCCGGGCAAGAG-3') }
\end{aligned}
$$

Alterations in PBP3 were investigated by sequencing the ftsI gene in all $\beta$-lactamase-negative isolates with MIC to ampicillin $\geq 1 \mu \mathrm{g} / \mathrm{ml}$ and in all $\beta$-lactamase-positive isolates 
with MIC to amoxicillin/clavulanic acid $\geq 2 \mu \mathrm{g} / \mathrm{ml}$. The $f t s I$ gene was first amplified by PCR using primers and conditions previously described by Cerquetti et al. [17], fts ${ }^{\text {frw }}$ (5'-GACG ATTTGGATAACCCATA-3'); positions 1197620 to 1197639 of the $H$. influenzae strain Rd sequence and $\mathrm{ftsI}^{\mathrm{rev}}$ (5'-CTGG ATAATTCTGTCTCAGA-3'); positions 1199884 to 1199865. To increase the sequence accuracy, we designed and used another set of primers: ftsI $2^{\text {frw }}$ (5'-GCGGATAA AGAACGAATTGC-3'); positions 1198212 to 1198232 of the $H$. influenzae strain Rd sequence and ftsI $2^{\text {rev }}\left(5^{\prime}\right.$-TCTC CTGCTTTTGGATCATTG-3'); positions 1199485 to 1199506. The sequenced products from amplification of the ftsI gene were analyzed and corrected using the CodonCode aligner software (http://www.codoncode.com/aligner). The amino acid substitutions from PBP3 protein sequences were investigated using clustalX version 2.0 [18]. Sequences were compared with those of $H$. influenzae RD KW20 (accession number NC_000907).

\section{Serotype determination using the slide agglutination test}

The slide agglutination test was performed on all 124 clinical isolates. It was performed on several colonies (pure culture) from overnight growth on a chocolate agar supplemented with polyvitex using Difco $H$. influenzae antisera (Becton Dickinson AG) according to the manufacturer's instructions.

\section{Results}

\section{Characteristics of patients and serotype $b$ distribution}

Among 124 isolates, 32 (25.8 \%) were invasive isolates and $92(74.2 \%)$ were respiratory tract isolates. The source of isolates were: 25 blood cultures, 5 pleural fluids, 29 sputa, 24 tracheal aspirates, 14 nasopharyngeal swabs, 8 eye swabs, 15 bronchoalveolar lavages, 2 middle-ear fluids, 1 cerebrospinal fluid, and 1 synovial fluid. Males accounted for $61 \%$ of the cases. Fifteen isolates were from pre-school children $(<5$ years old), 5 from older children (6-17 years old), 28 from adults (18-60 years old), and 76 from older patients ( $>60$ years old). The prevalence of encapsulated type b $H$. influenzae (Hib) among our collection was $4.8 \%$ (6 out of 124 ; Table 1$)$. Serotype b strains were isolated from 2 pre-school children (1 from blood culture, and 1 from a nasopharyngeal swab), 1 adult (bronchoalveolar lavage), and 3 older patients ( 2 from tracheal aspirates, and 1 from sputum).

\section{Antimicrobial susceptibility testing and $\beta$-lactamase production}

Table 2 shows the MIC50, MIC90, MIC range, and susceptibility categories of $\beta$-lactam antibiotics for the 124
H. influenzae isolates grouped into the four different groups (BLNAS, BLPAR, gBLNAR, and gBLPACR). Ampicillin resistance was found in $36 \%$ of isolates (45 out of 124; 22 were BLPAR, 19 were gBLNAR, and 4 were gBLPACR). All isolates were susceptible to azithromycin, levofloxacin, and moxifloxacin. For cotrimoxazole, $21 \%(n=26)$ of the isolates were nonsusceptible. For chloramphenicol, $3 \%$ (2 gBLPACR and 2 BLPAR) of the isolates were nonsusceptible (MIC $=8 \mathrm{mg} / \mathrm{l})$. The rate of resistance to amoxicillin/clavulanic acid was $9 \%$ (11 out of 124) using a EUCAST breakpoint and $0.8 \%(1$ out of $124, \mathrm{MIC}=12 \mu \mathrm{g} / \mathrm{ml})$ with a CLSI breakpoint. The most important discrepancies detected between the EUCAST and CLSI criteria were for cefuroxime; by current CLSI criteria, $94 \%$ of BLNAS, $54 \%$ of gBLNAR, $100 \%$ of BLPAR, and $25 \%$ of gBLPACR isolates were susceptible to cefuroxime. EUCAST has defined two different breakpoints for cefuroxime, intravenous and oral; according to the intravenous breakpoint, $71 \%$ of BLNAS, $14 \%$ of gBLNAR, $87 \%$ of BLPAR, and $0 \%$ of gBLPACR isolates were susceptible to cefuroxime. Fifteen BLNAS isolates were nonsusceptible to cefuroxime (MIC range: $2-12 \mu \mathrm{g} / \mathrm{ml}$ ), but still susceptible to ampicillin (MIC range: $0.75-1 \mu \mathrm{g} / \mathrm{ml}$ ). Piperacillin-tazobactam was highly potent $(\mathrm{MIC} 90=0.125 \mu \mathrm{g} / \mathrm{ml}$ ); MIC range, 0.016 to $0.25 \mu \mathrm{g} / \mathrm{ml} ; 100 \%$ susceptible by current CLSI clinical breakpoints. One gBLNAR isolate was resistant to ceftriaxone (MIC $=0.25 \mu \mathrm{g} / \mathrm{ml}$ ) according to current EUCAST clinical breakpoints.

\section{Genetic determinants of $\beta$-lactam resistance}

Twenty-six isolates identified, by the chromogenic cephalosporin assay using nitrocefin as a subtract, as $\beta$-lactamasepositive (25 BLPAR and 1 BLPACR) were resistant to ampicillin (MIC for ampicillin ranged from 1.5 to $256 \mu \mathrm{g} / \mathrm{ml}$ ). One BLPAR isolate was $\beta$-lactamase-positive, but remained susceptible to ampicillin (MIC $=0.38 \mu \mathrm{g} / \mathrm{ml}$ ). Screening of the 26 BLPAR and 1 BLPACR isolates for the presence of TEM

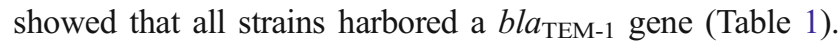
The sequence of ftsI encoding the transpeptidase region of PBP3 was determined in all $\beta$-lactamase-negative isolates with MIC to ampicillin $\geq 1 \mu \mathrm{g} / \mathrm{ml}$ and in all $\beta$-lactamasepositive isolates with MIC to amoxicillin/clavulanic acid $\geq 2 \mu \mathrm{g} / \mathrm{ml}$. Table 3 summarizes the amino acid changes observed, corresponding to $26 \%$ (32 out of 124) of the isolates (28 gBLNAR and 4 gBLPACR). The 9 BLNAS phenotype isolates (Table 3 ) found to possess mutations in the ftsI gene were re-designated as gBLNAR. The 3 BLPAR phenotype isolates found to possess both the $b l a_{\mathrm{TEM}-1}$ gene and mutations in the ftsI gene were re-designated as gBLPACR (strains: 22,34 , and 115). The most common amino acid substitutions were Asn526Lys (28 out of 32, 88 \%) and Val547Ile (28 out of $32,88 \%$ ), followed by Asp569Ser (23 out of 32, $72 \%$ ), Ala502Val (18 out of $32,56 \%$ ), Asp350Asn (18 out of 32 , 
Table 1 Ampicillin resistance classes of $124 \mathrm{H}$. influenzae isolates according to the type of specimen, age of patients, gender, and serotype b

\begin{tabular}{|c|c|c|c|c|c|c|c|c|c|c|c|c|}
\hline \multicolumn{11}{|l|}{ Phenotype } & \multicolumn{2}{|l|}{ Genotype } \\
\hline \multirow[t]{2}{*}{$\begin{array}{l}\text { Resistance } \\
\text { pattern }\end{array}$} & \multirow{2}{*}{$\begin{array}{l}\text { Total } \\
\text { isolates, } \\
n(\%)\end{array}$} & \multirow[t]{2}{*}{$\begin{array}{l}\text { Serotype } \\
\text { b, } n(\%)\end{array}$} & \multicolumn{4}{|c|}{$\begin{array}{l}\text { Ages group (number of } \\
\text { serotype b) }\end{array}$} & \multicolumn{2}{|c|}{ Gender } & \multicolumn{2}{|c|}{ Source of isolates } & \multirow[t]{2}{*}{ Type } & \multirow[t]{2}{*}{$\begin{array}{l}\text { Total } \\
\text { isolates. } n(\%)\end{array}$} \\
\hline & & & $\begin{array}{l}\leq 5 \\
\text { years }\end{array}$ & $\begin{array}{l}6-17 \\
\text { years }\end{array}$ & $\begin{array}{l}18-60 \\
\text { years }\end{array}$ & $\begin{array}{l}>60 \\
\text { years }\end{array}$ & Male & Female & $\begin{array}{l}\text { Invasive } \\
\text { isolates }\end{array}$ & $\begin{array}{l}\text { Respiratory } \\
\text { tract isolates }\end{array}$ & & \\
\hline BLNAS & $78(62.9)$ & $4(5.1)$ & $9(1)$ & 5 & $15(1)$ & $49(2)$ & 51 & 27 & 20 & 58 & BLNAS & $69(55.6)$ \\
\hline BLPAR & $26(21)$ & $2(7.7)$ & $4(1)$ & 0 & 7 & $15(1)$ & 13 & 13 & 7 & 19 & BLPAR (TEM-1) & $23(18.5)$ \\
\hline BLNAR & $19(15.3)$ & 0 & 2 & 0 & 6 & 11 & 12 & 7 & 5 & 14 & gBLNAR $^{\mathrm{a}}$ & $28(22.6)$ \\
\hline BLPACR & $1(0.8)$ & 0 & 0 & 0 & 0 & 1 & 0 & 1 & 0 & 1 & gBLPACR (TEM-1) & $4(3.3)$ \\
\hline Total number & $124(100.0)$ & $6(4.8)$ & $15(2)$ & $5(0)$ & $28(1)$ & $76(3)$ & 76 & 48 & 32 & 92 & & $124(100.0)$ \\
\hline
\end{tabular}

$B L N A S \beta$-lactamase-negative, ampicillin-susceptible, BLPAR $\beta$-lactamase-positive, ampicillin-resistant, $B L N A R \beta$-lactamase-negative, ampicillin-resistant $g B L N A R \beta$-lactamase-negative with amino acid substitution in the PBP3 BLPACR $\beta$-lactamase-positive, amoxicillin/clavulanic acid-resistant, gBLPACR $\beta$-lactamase-producing (TEM-1) with amino acid substitution in the PBP3

${ }^{a} 9$ BLNAS phenotype isolates (ampicillin MIC $=1 \mu \mathrm{g} / \mathrm{ml}$ ) found to possess mutations in the $f$ ts $I$ gene were re-designated as genomic BLNAR (gBLNAR)

${ }^{\mathrm{b}} 3$ BLPAR phenotype isolates (amoxicillin/clavulanic acid MIC $=2 \mu \mathrm{g} / \mathrm{ml}$ ) found to possess both the blaTEM-1 gene and mutations in the $f t s I$ gene were re-designated as genomic BLPACR (gBLPACR)

$56 \%$ ), Met 377 Ile (15 out of $32,47 \%$ ), Ile449Val ( 7 out of 32 , $22 \%$ ), and Arg517His ( 2 out of 32, $6.3 \%$ ). The patterns observed were classified into groups II and III-like, according to the criteria of Dabernat et al. [19] and Garcia-Cobos et al. [20], and a miscellaneous (M) group, according to the data from this study. Group II included 26 isolates subdivided into four subgroups:
1. Two isolates belonged to the subgroup IIa (1 isolate with Asn526Lys, Val547IIe, Asp569Ser substitutions), and another isolate with Asn526Lys and Asp350Asn substitutions)

2. Seventeen isolates were classified as subgroup IIb, defined by Asn526Lys and Ala502Val substitutions (those isolates also presented the substitutions Asp350Asn and

Table 2 Susceptibilities of $124 \mathrm{H}$. influenzae isolates according to European Committee on Antimicrobial Susceptibility Testing (EUCAST) and Clinical and Laboratory Standards Institute (CLSI) clinical breakpoints to four $\beta$-lactam antibiotics

\begin{tabular}{|c|c|c|c|c|c|c|c|c|c|c|c|}
\hline \multirow[t]{2}{*}{ Antibiotic } & \multirow[t]{2}{*}{ Phenotype/genotype } & \multirow[t]{2}{*}{ Total isolates } & \multicolumn{3}{|c|}{$\mathrm{MIC} \mu \mathrm{g} / \mathrm{ml}$} & \multicolumn{3}{|c|}{$\begin{array}{l}\text { EUCAST clinical } \\
\text { breakpoints ( } \% \text { isolates) }\end{array}$} & \multicolumn{3}{|c|}{$\begin{array}{l}\text { CLSI clinical breakpoints } \\
\text { (\% isolates) }\end{array}$} \\
\hline & & & MIC 50 & MIC 90 & MIC range & $\mathrm{S}$ & I & $\mathrm{R}$ & $\mathrm{S}$ & I & $\mathrm{R}$ \\
\hline \multirow[t]{4}{*}{ Ampicillin } & BLNAS & 69 & 0.38 & 0.75 & $0.064-1$ & 100 & & 0 & 100 & 0 & 0 \\
\hline & BLPAR & 23 & 12 & 256 & $0.38-256$ & 4 & & 96 & 4 & 22 & 74 \\
\hline & gBLNAR & 28 & 1.5 & 2 & $1-3$ & 32 & & 68 & 32 & 68 & 0 \\
\hline & gBLPACR & 4 & 128 & 256 & $24-256$ & 0 & & 100 & 0 & 0 & 100 \\
\hline \multirow{4}{*}{$\begin{array}{l}\text { Amoxicillin / clavulanic } \\
\text { acid }\end{array}$} & BLNAS & 69 & 0.5 & 1 & $0.094-2$ & 100 & & 0 & 100 & 0 & 0 \\
\hline & BLPAR & 23 & 1 & 1.5 & $0.38-1.5$ & 100 & & 0 & 100 & 0 & 0 \\
\hline & gBLNAR & 28 & 2 & 4 & $0.75-4$ & 64 & & 36 & 100 & 0 & 0 \\
\hline & gBLPACR & 4 & 2 & 2 & $2-12$ & 75 & & 15 & 75 & 0 & 15 \\
\hline \multirow[t]{4}{*}{ Cefuroxime } & BLNAS & 69 & 1 & 2 & $0.19-12$ & 71 & 20 & 9 & 94 & 6 & 0 \\
\hline & BLPAR & 23 & 0.75 & 1.5 & $0.25-3$ & 87 & 8 & 5 & 100 & 0 & 0 \\
\hline & gBLNAR & 28 & 4 & 24 & $0.25-256$ & 14 & 14 & 72 & 54 & 25 & 21 \\
\hline & gBLPACR & 4 & 6 & 6 & $2-24$ & 0 & 25 & 75 & 25 & 50 & 25 \\
\hline \multirow[t]{4}{*}{ Piperacillin/tazobactam } & BLNAS & 69 & 0.023 & 0.125 & $0.016-0.19$ & & & & 100 & 0 & 0 \\
\hline & BLPAR & 23 & 0.032 & 0.094 & $0.016-0.5$ & & & & 100 & 0 & 0 \\
\hline & gBLNAR & 28 & 0.064 & 0.19 & $0.016-0.25$ & & & & 100 & 0 & 0 \\
\hline & gBLPACR & 4 & 0.064 & 0.094 & $0.064-0.25$ & & & & 100 & 0 & 0 \\
\hline
\end{tabular}




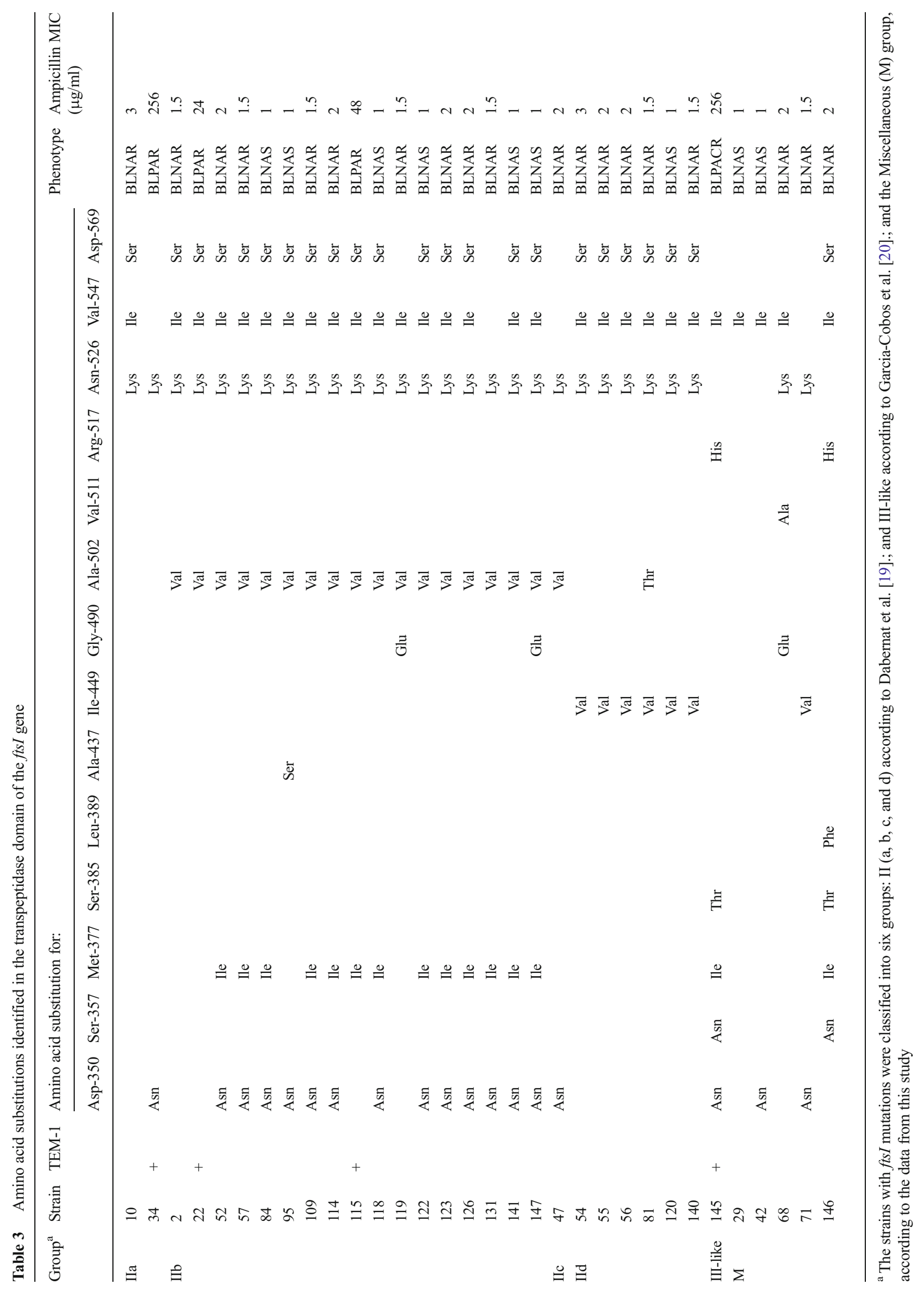


Met377Ile, and two of them had Gly490Glu, and one of them had also Ala437Ser)

3. One isolate was classified as subgroup IIc, characterized by Asn526Lys, Ala502Val, and Asp350Asn)

4. Six isolates were classified as subgroup IId, characterized by Asn526Lys, Ile449Val, and one of them had also Ala502Thr)

One isolate belonged to the group III-like (previously described by Garcia-Cobos et al. [20]). Five patterns (5 isolates) were characterized and classified into the miscellaneous group: one of them ( 1 isolate) has already been described by Garcia-Cobos et al. [20], while the remaining 4 were determined for the first time in this study (1 isolate with Asp350Asn and Val547Ile; 1 isolate with only Val547Ile; 1 isolate with Asp350Asn, Ile449Val, Asn526Lys, and Val547Ile; and 1 isolate with Ser357Asn, Met377lle, Ser385Thr, Leu389Phe, Arg517His, Val547Ile, and Asp569Ser). Three isolates belonging to the miscellaneous group were nonsusceptible to ampicillin (MIC range: $1.5-2 \mu \mathrm{g} / \mathrm{ml}$ ) and to cefuroxime (MIC range: $8-$ $64 \mu \mathrm{g} / \mathrm{ml}$ ). One isolate (number 146) with Ser357Asn, Met377Ile, Ser385Thr, Leu389Phe, and Arg517His, was resistant according to EUCAST criteria to ampicillin ( $\mathrm{MIC}=$ $2 \mu \mathrm{g} / \mathrm{ml}$ ), to amoxicillin/clavulanic acid (MIC $=4 \mu \mathrm{g} / \mathrm{ml}$ ), and to ceftriaxone $(\mathrm{MIC}=0.25 \mu \mathrm{g} / \mathrm{ml})$. Nine of the 28 gBLNAR isolates were susceptible to ampicillin (BLNAS phenotype; MIC $=1 \mu \mathrm{g} / \mathrm{ml}$ ), but 6 out of 9 were nonsusceptible to cefuroxime (MIC range: 3-6 $\mu \mathrm{g} / \mathrm{ml}$ ), suggesting that these mutations might have been involved in decreased $\beta$-lactam susceptibility. Among gBLNAR strains, higher ampicillin MICs have been previously associated with group III-like isolates because of the unique patterns of amino acid substitutions exhibited by these strains [20]. However, in this study one strain belonging to the III-like group showed a particularly high ampicillin MIC $(256 \mu \mathrm{g} / \mathrm{ml})$, was resistant to amoxicillin/ clavulanic acid ( $\mathrm{MIC}=12 \mu \mathrm{g} / \mathrm{ml})$ and cefuroxime (MIC $=6$ $\mu \mathrm{g} / \mathrm{ml})$, and harbored a $b l a_{\mathrm{TEM}-1}$ gene (gPLPACR). In most of the strains belonging to the different subgroups of group II, we observed an association between reduced susceptibility to ampicillin and the presence of Val547Ile and Asp569Ser substitutions. The gBLNAR mechanism was the most prevalent in this study ( 28 out of $124 ; 23 \%$ ), exceeding the proportion of isolates that produced $\beta$-lactamase ( 23 out of $124 ; 19 \%$ ). Invasive gBLNAR isolates belonged to the following groups: 1 strain to group IIa, 3 strains to group IIb, and 1 strain to group IId. Of the 23 BLPAR isolates, 7 were invasive (Table 1). The 3 BLPAR phenotype isolates found to possess both the $b l a_{\mathrm{TEM}-1}$ gene and mutations in the ftsI gene (gBLPACR) were found in the following subgroups: IIa (1 isolate; ampicillin $\mathrm{MIC}=256 \mu \mathrm{g} / \mathrm{ml})$ and $\mathrm{IIb}(2$ isolates: ampicillin MICs $=24$ and $48 \mu \mathrm{g} / \mathrm{ml}$ ). The amoxicillin/clavulanic acid MIC was $2 \mu \mathrm{g} /$ $\mathrm{ml}$ for all three of these isolates, which were resistant to cefuroxime (MICs $=2,6$, and $24 \mu \mathrm{g} / \mathrm{ml}$ ).

\section{Disk diffusion versus Etest method}

Table 4 shows the susceptibility categories of ampicillin and amoxicillin/clavulanic acid for the 69 BLNAS and 9 gBLNAR isolates according to the disk diffusion and Etest methods (using EUCAST breakpoints). Ampicillin and amoxicillin/clavulanic acid resistance were found in 26 out of 69 BLNAS ( $38 \%$ ) and in 7 out of 9 gBLNAR (78 \%) using the disk diffusion method. For ampicillin the inhibition zones ranged between 12 and $19 \mathrm{~mm}$. For amoxicillin/clavulanic acid the inhibition zones ranged between 13 and $17 \mathrm{~mm}$. Read zone edges in Mueller-Hinton agar $+5 \%$ defibrinated horse blood and $20 \mathrm{mg} / \mathrm{L} \beta$-NAD (incubated with $5 \% \mathrm{CO}_{2}$, $35 \pm 1{ }^{\circ} \mathrm{C}, 18 \pm 2 \mathrm{~h}$ ) as the point showing absence of growth viewed from the front of the plate with the lid removed and with reflected light. The Etest method revealed a high degree of susceptibility for all 78 isolates (Table 4). The European Committee on Antimicrobial Susceptibility Testing recommend using benzylpenicillin disks $(1 \mathrm{U})$ in Mueller-Hinton agar $+5 \%$ defibrinated horse blood and $20 \mu \mathrm{g} / \mathrm{ml} \beta$-NAD to

Table 4 Comparison of the susceptibility of $78 \beta$-lactamase-negative $H$. influenzae isolates (69 BLNAS and 9 gBLNAR) with ampicillin and amoxicillin/clavulanic acid determined using the disk diffusion and Etest methods

\begin{tabular}{|c|c|c|c|c|c|c|c|c|c|c|}
\hline \multirow[t]{3}{*}{ Antibiotic } & \multirow[t]{3}{*}{ Phenotype/genotype } & \multirow[t]{3}{*}{ Total isolates } & \multicolumn{8}{|c|}{ Susceptibility category ${ }^{\mathrm{b}}$} \\
\hline & & & \multicolumn{3}{|c|}{ Disk diffusion (mm) } & \multicolumn{5}{|c|}{ Etest method $(\mu \mathrm{g} / \mathrm{ml})$} \\
\hline & & & $\mathrm{R}(\%)$ & $\mathrm{S}(\%)$ & $\begin{array}{l}\text { Inhibition } \\
\text { zone range }\end{array}$ & MIC 50 & MIC 90 & MIC range & $\mathrm{R}(\%)$ & S (\%) \\
\hline \multirow[t]{2}{*}{ Ampicillin } & BLNAS & 69 & $26 / 69(38)$ & $43 / 69(62)$ & $12-19$ & 0.38 & 0.75 & $0.125-1$ & 0 & 100 \\
\hline & gBLNAR $^{\mathrm{a}}$ & 9 & $7 / 9(78)$ & $2 / 9(22)$ & $15-17$ & 1 & 1 & 1 & 0 & 100 \\
\hline \multirow{2}{*}{$\begin{array}{l}\text { Amoxicillin/clavulanic } \\
\text { acid }\end{array}$} & BLNAS & 69 & $26 / 69(38)$ & $43 / 69(62)$ & $13-17$ & 0.5 & 1.5 & $0.125-2$ & 0 & 100 \\
\hline & gBLNAR $^{\mathrm{a}}$ & 9 & $7 / 9(78)$ & $2 / 9(22)$ & $14-16$ & 1.5 & 2 & $0.75-2$ & 0 & 100 \\
\hline
\end{tabular}

${ }^{a} 9$ BLNAS phenotype isolates (ampicillin MIC $=1 \mu \mathrm{g} / \mathrm{ml}$ ) found to possess mutations in the $\mathrm{fts} I$ gene were re-designated as gBLNAR

${ }^{\mathrm{b}}$ The interpretative criteria were based on the EUCAST clinical breakpoints 
screen for $\beta$-lactam resistance. All 78 isolates were fully susceptible to penicillin $(1 \mathrm{U})$ with an inhibition zone $>12 \mathrm{~mm}$. Preliminary results from our laboratory therefore reflect an important increase in $\beta$-lactam resistance in $H$. influenzae when ampicillin and amoxicillin/clavulanic acid were tested using disk diffusion. For these antibiotics the disk diffusion and Etest methods are not entirely interchangeable.

\section{Discussion}

Over the past decade many countries have documented a rapid increase in the prevalence of BLNAR non-typeable H. influenzae (NTHi) isolates, particularly among respiratory tract isolates [21]. Genotypically defined BLNAR (gBLNAR) isolates account for $15-30 \%$ of all NTHi isolates in Europe [22]. The detection of this phenotype is clinically relevant, as these strains have lower responsiveness to $\beta$-lactam and are not affected by the addition of clavulanic acid. Note, however, that the gBLNAR strains with ampicillin $\mathrm{MIC}=1 \mathrm{mg} / \mathrm{L}$ are considered resistant according to neither the current CLSI breakpoints nor the European Committee on Antimicrobial Susceptibility Testing (EUCAST) guidelines. Of the 78 BLNAS isolates analyzed in this study, 14 strains had ampicillin $\mathrm{MIC}=1 \mathrm{mg} / \mathrm{L}$. Of these 14 strains, 9 out of $14(65 \%)$ were revealed to possess mutations in the ftsI gene and were therefore re-designated as gBLNAR. Six isolates belonged to the subgroup IIb, 1 isolate belonged to the subgroup IId, and 2 isolates belonged to the miscellaneous (M) group. Our findings are in agreement with those of other studies that have detected mutations in the ftsI gene of these strains, resulting in PBP3 substitutions responsible for the decreased susceptibility to ampicillin $[2,4]$.

Since 2001, the genetic basis of BLNAR was clarified and attributed to the presence of either an Asn526Lys or an Arg517His substitution in PBP3, encoded by de novo point mutations in the ftsI gene [17]. In our study an important diversity in PBP3 mutations was found and were categorized into six main genotypic patterns (groups/subgroups) based on the amino acid substitutions. Among our strains, the predominance of group II, with the most common substitution Asn526 to Lys, is in agreement with previous studies $[2,8,9,12]$. The amino acid substitutions at positions Met377, Ser385, and Leu389 around the SSN motif, which decrease susceptibility to cephalosporins, were detected among our BLNAR strains. Our study confirms the correlation between BLNAR-defining amino acid substitutions and the ampicillin resistance phenotype established in earlier studies $[1,2]$. However, it appears obvious that mechanisms other than PBP-3 mutations or $\beta$-lactamase production contribute to $\beta$ lactam resistance in $H$. influenzae. Among potential mechanisms, disrupted repression of the acrR efflux pump or alterations in outer membrane protein 2 (OMP2) have been suggested $[8,23]$.
The BLPACR strains, which have two mechanisms of antimicrobial resistance, including $\beta$-lactamase production and mutations in the ftsI gene, show resistance against many $\beta$ lactam. High detection rates of BLPACR strains have been reported in Spain (22\%) from 2005 to 2007 and in some cases clonal dissemination of the BLPACR strains has been observed [12]. In this study, of the 124 isolates analyzed, 4 were gBLPACR ( 3 BLPAR and 1 BLPACR). These isolates belonged to the following groups: IIa, IIb, and III-like. The 3 BLPAR phenotype isolates found to possess both the $b l a_{\mathrm{TEM}-1}$ gene and mutations in the $f t s I$ gene, showed particularly high ampicillin MICs $(24,48$, and $256 \mu \mathrm{g} / \mathrm{ml})$ and were resistant to cefuroxime ( $\mathrm{MICs}=6$, and $24 \mu \mathrm{g} / \mathrm{ml}$ ), but still susceptible to amoxicillin/clavulanic acid (MIC $=2 \mu \mathrm{g} / \mathrm{ml}$ ). In this study, 6 out of 32 gBLNAR (19\%), 19 out of 32 BLNAS (59 \%), and 7 out of 32 BLPAR (22\%) strains were detected in invasive isolates. gBLPACR strains were not found in invasive isolates. All 32 invasive isolates were susceptible to ceftriaxone, which has been the first-line antibiotic for the treatment of bacterial meningitis. In this study, $29 \%$ of BLNAS, $13 \%$ of BLPAR, $86 \%$ of gBLNAR, and $100 \%$ of gBLNACR were nonsusceptible to cefuroxime (using EUCAST breakpoints). Amoxicillin/clavulanic acid and oral cephalosporins are frequently used for the treatment of respiratory tract infections in our country. It is therefore necessary to monitor the increased occurrence and spread of BLNAR and BLPACR isolates in the community. Among the $\beta$-lactam antibiotics tested in this study, piperacillin-tazobactam clearly exhibited very strong activities against all classes of H. influenzae isolates analyzed (Table 2). All 124 isolates were susceptible to piperacillin-tazobactam according to the current CLSI breakpoint. Piperacillin-tazobactam, previously considered to be inactive against BLNAR H. influenzae owing to limited data, has now been found to have great potency against these strains and should be assigned accurate susceptibility breakpoints [24]. The European Committee on Antimicrobial Susceptibility Testing does not yet provide any breakpoint for piperacillin-tazobactam interpretation of MICs and zone diameters.

Invasive infections due to $H$. influenzae are usually treated with $\beta$-lactam antibiotics, including aminopenicillins or cephalosporins. Interestingly, BLNAR isolates with PBP3 modifications show decreased susceptibility to both aminopenicillins and cephalosporins. Of the 28 gBLNAR isolates, 5 were invasive $(18 \%)$. Three strains were isolated from blood culture. One isolate was from a pre-school child (male, 3 years old), 1 from a young adult (female, 21 years old), and 1 from an adult (male, 74 years old).

For many years, the screening method for the identification of $\beta$-lactam-resistant $H$. influenzae has been disk diffusion testing for penicillin and cefaclor followed by a nitrocefin $\beta$ lactamase test. Even though penicillin is rarely an alternative for treatment of $H$. influenzae infections, experience suggests 
that this method might be suitable for resistance surveillance, allowing for sensitive monitoring of $\beta$-lactam resistance. Furthermore, the reliability of the disk diffusion method for defining precise levels of $\beta$-lactam resistance in $H$. influenzae has been questioned. In this study, ampicillin and amoxicillin/ clavulanic acid resistance were found in 26 out of 69 BLNAS (38\%) and in 7 out of 9 gBLNAR ( $78 \%$ ) using disk diffusion. The Etest method indicated a high degree of susceptibility for all these 78 isolates. However, as a primary screening method for resistance in clinical isolates, when followed by a detailed examination (MIC determination and ftsI gene sequencing), the disk diffusion method was considered suitable for the detection of low-level $\beta$-lactamase-negative ampicillin-resistant (low-BLNAR) H. influenzae [25]. Etest remains a reliable method for defining precise levels of $\beta$-lactam resistance in $H$. influenzae. In a study conducted by Cerquetti et al. [17], the authors were able to detect two NTHI isolates showing heterogeneous resistance to imipenem by using the Etest. Checking for $\beta$ lactamase alone is performed for clinical isolates of $H$. influenzae, and antimicrobial susceptibilities are not accurately determined in several laboratories. This may result in the lack of detection of BLNAR and BLPACR. The gold standard method of characterizing strains exhibiting this non-enzymatic mechanism of resistance is the sequencing of the ftsI gene.

In summary, this was a retrospective study of serotype $\mathrm{b}$ distribution, phenotypic antibiotic resistance, and ampicillin resistance mechanisms of 124 invasive and respiratory tract $H$. influenzae isolates. To the best of our knowledge, a similar microbiological characterization of clinical H. influenzae strains has not been previously done in Switzerland. In this study we confirmed the low proportion of $\beta$-lactamase-positive ampicillin-resistant invasive serotype $b$ isolates in our institution. The emergence of BLNAR and BLPACR strains among respiratory tract isolates may have implications for the treatment of respiratory tract $H$. influenzae infections. The detection of this phenotype is clinically relevant, as these strains have lower responsiveness to $\beta$-lactam and are not targeted by clavulanic acid. Continued monitoring of $\beta$-lactam susceptibility and the characterization of both invasive and respiratory $H$. influenzae isolates are necessary to observe changes in the microbiology and epidemiology of this important pathogen that could lead to clinical failure when treated with empirical antibiotics.

\section{Transparency declarations Nothing to declare.}

Conflict of Interest The authors declare that they have no conflict of interest.

\section{References}

1. Skaare D, Anthonisen IL, Kahlmeter G, Matuschek E, Natas OB, Steinbakk M, Sundsfjord A, Kristiansen BE (2014) Emergence of clonally related multidrug resistant Haemophilus influenzae with penicillin-binding protein 3-mediated resistance to extendedspectrum cephalosporins, Norway, 2006 to 2013. Euro Surveill 19(49)

2. Barbosa AR, Giufre M, Cerquetti M, Bajanca-Lavado MP (2011) Polymorphism in ftsI gene and \{beta\}-lactam susceptibility in Portuguese Haemophilus influenzae strains: clonal dissemination of beta-lactamase-positive isolates with decreased susceptibility to amoxicillin/clavulanic acid. J Antimicrob Chemother 66(4):788796

3. Setchanova LP, Kostyanev T, Markovska R, Miloshev G, Mitov IG (2013) Serotypes, antimicrobial susceptibility, and beta-lactam resistance mechanisms of clinical Haemophilus influenzae isolates from Bulgaria in a pre-vaccination period. Scand J Infect Dis 45(2):81-87

4. Bajanca-Lavado MP, Simoes AS, Betencourt CR, Sa-Leao R (2014) Characteristics of Haemophilus influenzae invasive isolates from Portugal following routine childhood vaccination against $\mathrm{H}$. influenzae serotype b (2002-2010). Eur J Clin Microbiol Infect Dis 33(4):603-610

5. Park C, Kim KH, Shin NY, Byun JH, Kwon EY, Lee JW, Kwon HJ, Choi EY, Lee DG, Sohn WY, Kang JH (2013) Genetic diversity of the ftsI gene in beta-lactamase-nonproducing ampicillin-resistant and beta-lactamase-producing amoxicillin-/clavulanic acidresistant nasopharyngeal Haemophilus influenzae strains isolated from children in South Korea. Microb Drug Resist 19(3):224-230

6. Skaare D, Anthonisen IL, Caugant DA, Jenkins A, Steinbakk M, Strand L, Sundsfjord A, Tveten Y, Kristiansen BE (2014) Multilocus sequence typing and ftsI sequencing: a powerful tool for surveillance of penicillin-binding protein 3-mediated beta-lactam resistance in nontypeable Haemophilus influenzae. BMC Microbiol 14:131

7. Hasegawa K, Yamamoto K, Chiba N, Kobayashi R, Nagai K, Jacobs MR, Appelbaum PC, Sunakawa K, Ubukata K (2003) Diversity of ampicillin-resistance genes in Haemophilus influenzae in Japan and the United States. Microb Drug Resist 9(1):39-46

8. Resman F, Ristovski M, Forsgren A, Kaijser B, Kronvall G, Medstrand P, Melander E, Odenholt I, Riesbeck K (2012) Increase of beta-lactam-resistant invasive Haemophilus influenzae in Sweden, 1997 to 2010. Antimicrob Agents Chemother 56(8): $4408-4415$

9. Puig C, Calatayud L, Marti S, Tubau F, Garcia-Vidal C, Carratala J, Linares J, Ardanuy C (2013) Molecular epidemiology of nontypeable Haemophilus influenzae causing communityacquired pneumonia in adults. PLoS One 8(12), e82515

10. Kishii K, Chiba N, Morozumi M, Hamano-Hasegawa K, Kurokawa I, Masaki J, Ubukata K (2010) Diverse mutations in the ftsI gene in ampicillin-resistant Haemophilus influenzae isolates from pediatric patients with acute otitis media. J Infect Chemother : Off J Jpn Soc Chemother 16(2):87-93

11. Hoshino T, Sato Y, Toyonaga Y, Hanaki H, Sunakawa K (2013) Nationwide survey of the development of drug resistance in the pediatric field in 2007 and 2010: drug sensitivity of Haemophilus influenzae in Japan (second report). J Infect Chemother : Off J Jpn Soc Chemother 19(3):495-503

12. Dabernat H, Delmas C (2012) Epidemiology and evolution of antibiotic resistance of Haemophilus influenzae in children 5 years of age or less in France, 2001-2008: a retrospective database analysis. Eur J Clin Microbiol Infect Dis 31(10):2745-2753 
13. Ladhani S, Slack MP, Heath PT, von Gottberg A, Chandra M, Ramsay ME (2010) Invasive Haemophilus influenzae disease, Europe, 1996-2006. Emerg Infect Dis 16(3):455-463

14. Bruin JP, Kostrzewa M, van der Ende A, Badoux P, Jansen R, Boers SA, Diederen BM (2014) Identification of Haemophilus influenzae and Haemophilus haemolyticus by matrix-assisted laser desorption ionization-time of flight mass spectrometry. Eur J Clin Microbiol Infect Dis 33(2):279-284

15. Cherkaoui A, Hibbs J, Emonet S, Tangomo M, Girard M, Francois P, Schrenzel J (2010) Comparison of two matrix-assisted laser desorption ionization-time of flight mass spectrometry methods with conventional phenotypic identification for routine identification of bacteria to the species level. J Clin Microbiol 48(4):1169-1175

16. Frickmann H, Christner M, Donat M, Berger A, Essig A, Podbielski A, Hagen RM, Poppert S (2013) Rapid discrimination of Haemophilus influenzae, $\mathrm{H}$. parainfluenzae, and $\mathrm{H}$. haemolyticus by fluorescence in situ hybridization (FISH) and two matrixassisted laser-desorption-ionization time-of-flight mass spectrometry (MALDI-TOF-MS) platforms. PLoS One 8(4), e63222

17. Cerquetti M, Giufre M, Cardines R, Mastrantonio P (2007) First characterization of heterogeneous resistance to imipenem in invasive nontypeable Haemophilus influenzae isolates. Antimicrob Agents Chemother 51(9):3155-3161

18. Larkin MA, Blackshields G, Brown NP, Chenna R, McGettigan PA, McWilliam H, Valentin F, Wallace IM, Wilm A, Lopez R, Thompson JD, Gibson TJ, Higgins DG (2007) Clustal W and Clustal X version 2.0. Bioinformatics 23(21):2947-2948

19. Dabernat H, Delmas C, Seguy M, Pelissier R, Faucon G, Bennamani S, Pasquier C (2002) Diversity of beta-lactam resistance-conferring amino acid substitutions in penicillin-binding protein 3 of Haemophilus influenzae. Antimicrob Agents Chemother 46(7):2208-2218
20. Garcia-Cobos S, Campos J, Lazaro E, Roman F, Cercenado E, Garcia-Rey C, Perez-Vazquez M, Oteo J, de Abajo F (2007) Ampicillin-resistant non-beta-lactamase-producing Haemophilus influenzae in Spain: recent emergence of clonal isolates with increased resistance to cefotaxime and cefixime. Antimicrob Agents Chemother 51(7):2564-2573

21. Tristram S, Jacobs MR, Appelbaum PC (2007) Antimicrobial resistance in Haemophilus influenzae. Clin Microbiol Rev 20(2):368-389

22. Witherden EA, Bajanca-Lavado MP, Tristram SG, Nunes A (2014) Role of inter-species recombination of the ftsI gene in the dissemination of altered penicillin-binding-protein-3-mediated resistance in Haemophilus influenzae and Haemophilus haemolyticus. J Antimicrob Chemother 69(6):1501-1509

23. Kaczmarek FS, Gootz TD, Dib-Hajj F, Shang W, Hallowell S, Cronan M (2004) Genetic and molecular characterization of betalactamase-negative ampicillin-resistant Haemophilus influenzae with unusually high resistance to ampicillin. Antimicrob Agents Chemother 48(5):1630-1639

24. Hirakata Y, Ohmori K, Mikuriya M, Saika T, Matsuzaki K, Hasegawa M, Hatta M, Yamamoto N, Kunishima H, Yano H, Kitagawa M, Arai K, Kawakami K, Kobayashi I, Jones RN, Kohno S, Yamaguchi K, Kaku M (2009) Antimicrobial activities of piperacillin-tazobactam against Haemophilus influenzae isolates, including beta-lactamase-negative ampicillin-resistant and beta-lactamase-positive amoxicillin-clavulanate-resistant isolates, and mutations in their quinolone resistance-determining regions. Antimicrob Agents Chemother 53(10):4225-4230

25. Norskov-Lauritsen N, Ridderberg W, Erikstrup LT, Fuursted K (2011) Evaluation of disk diffusion methods to detect low-level beta-lactamase-negative ampicillin-resistant Haemophilus influenzae. APMIS 119(6):385-392 